\title{
Evaluating the Role of Perceived Stress on the Likelihood of Having a Non - Fatal Acute Coronary Syndrome: A Case-Control Study
}

\author{
Alexia Katsarou ${ }^{\mathrm{a}}$, Filippos Triposkiadis ${ }^{\mathrm{a}}$, John Skoularigis ${ }^{\mathrm{a}}$, Emmanuela Griva ${ }^{\mathrm{b}}$, George Neroutsos ${ }^{\mathrm{b}}$, Georgios \\ Karayannis $^{\mathrm{a}}$, Charalabos Papageorgiou ${ }^{\mathrm{c}}$ and Demosthenes Panagiotakos ${ }^{*}$,
}

${ }^{a}$ Department of Cardiology, School of Medicine, University of Thessaly, Larissa, Greece

${ }^{b}$ Department of Nutrition and Dietetics, Harokopio University, Athens, Greece

${ }^{c}$ First Department of Psychiatry, Eginition Hospital, School of Medicine, University of Athens, Athens, Greece

\begin{abstract}
Objectives: The aim of the current study was to evaluate the independent role of perceived stress, measured by the PSS-14, on the likelihood of having acute coronary syndrome (ACS).

Methods: This is a case-control study with individual matching by age and sex. During 2010-2012, 250 consecutive patients $(60 \pm 11$ years, $78 \%$ men) with a first ACS and 250 population-based, control subjects $(60 \pm 8.6$ years, $77.6 \%$ men), were enrolled. Perceived stress levels were evaluated with the PSS-14 scale, depression status was assessed with the Zung Depression Rating Scale, anxiety status with the STAI scale and adherence to the Mediterranean diet was assessed by the MedDietScore.

Results: Higher perceived stress was associated with increased likelihood of having an ACS, after adjusting for various factors (OR=1.15, \%CI 1.11, 1.18). STAI and ZUNG scale were positively associated with the likelihood of having an ACS (OR: $1.27 \%$ CI 1.20-1.34, p<0.001 and OR: $1.49 \%$ CI 1.36-1.63, $<<0.001$ respectively). Stratified analysis by sex showed a greater impact of perceived stress in men, compared with women (Wald test value $45.65 v s$ 18.56, respectively). When stratifying by depression levels, the effect of perceived stress on ACS was not significant among depressed individuals. When stratifying by level of anxiety, higher odds of having an ACS was found in the low anxiety group (OR: 1.129, \%CI 1.047$1.218)$

Conclusion: Perceived stress appears as an independent ACS risk factor, although no causal relationship can be extracted due to the nature of the study. Early recognition and treatment of perceived stress may lead to ACS risk reduction.
\end{abstract}

Keywords: Acute coronary syndrome, anxiety, depression, Mediterranean diet, perceived stress, psychosocial.

\section{INTRODUCTION}

Cardiovascular disease (CVD) continues to be the prominent cause of death in Europe and developing countries and presents the primary somatic cause of loss of productivity [1]. Psychosocial factors and their contribution to CVD pathogenesis and incidence have gained great attention [1,2]. Stress has been associated with increased coronary heart disease (CHD) incidence and prevalence $[3,4]$. Particularly, several types of stress (i.e. work or marital stress) have served as well-defined constructs in the field of research regarding the role of psychosocial stress in CVD development [5, 6]. However, in research, stress is not always measured according to an integrated and discrete theoretical framework or constructs (i.e. work or marital stress). Consequently, it is possible that only several stress

*Address correspondence to this author at the 46 Paleon Polemiston St., 16674, Glyfada, Greece; Tel: +30 210 9603116; Fax: +30 210 9600719;

E-mail: d.b.panagiotakos@usa.net parameters are estimated, a fact that may hinder the exact evaluation of the independent role of the concept of stress on CVD incidence.

Interestingly, perceived stress has also emerged as a promising psychosocial CVD risk factor [7, 8]. Perceived stress presents a global and comprehensive stress construct that refers to the transactional process between the individual and the environment and seems to be associated with a moderately increased risk of CVD incidence. In a recent meta-analysis, including studies that evaluated prospectively the role of perceived stress in CVD incidence, high perceived stress was associated with a $27 \%$ increase in CHD risk, a percentage reported as equivalent of a $50 \mathrm{mg} / \mathrm{dl}$ increase in low-density lipoprotein, a $2.7 / 1.4 \mathrm{mmHg}$ increase in systolic/diastolic blood pressure, and 5 more cigarettes/day [9]. In most studies, the relationship observed between perceived stress and CVD incidence emerges as causal and, thus, perceived stress acts clearly as an independent CVD risk factor $[8,9]$. However, to better 
clarify the nature of the role of perceived stress in CVD development and incidence further research is required.

On this basis, perceived stress seems to be an important chronic psychosocial CVD risk factor, but yet not fully established and understood. Thus, the aim of the current study was to evaluate the independent role of perceived stress, measured by the PSS-14 scale, on the likelihood of developing acute coronary syndromes (ACS).

\section{MATERIALS AND METHODS}

\section{Design}

This is a case-control study with individual (one-to-one) matching by age (within \pm 3 years) and sex, and in accordance to a larger epidemiological protocol [10].

\section{Bioethics}

The study was approved by the Ethics Committee of the University Hospital of Thessaly (Larissa) and was carried out in accordance with the Declaration of Helsinki (1989) of the World Medical Association. Before collecting any information, participants were informed about the aims and procedures of the study and provided their signed consent.

\section{Sampling Procedure}

From August 2010 to September 2012, 250 patients with a first ACS event and without any suspicion of previous CVD, that attended the cardiology, pathology clinics or the emergency units of 2 major General Hospitals, in Greece agreed to participate (participation rate $=75 \%$ ). Regarding the ACS patients, clinical symptoms were evaluated at hospital entry and a 12-lead electrocardiogram was performed. Evidence of myocardial cell death was assessed with blood tests and measurement of the levels of troponin I and the MB fraction of creatine kinase (CK) [11]. Two hundred and fifty control subjects, age-sex matched one-byone with ACS patients, were selected concurrently with the patients on a volunteer, population basis, and from the same region of the patients. Controls were without any clinical symptoms or suspicions of CVD in their medical history, as assessed by a physician. Subjects with chronic neoplastic disease or chronic inflammatory disease, as well as individuals with recent changes in their dietary habits, were not enrolled.

Based on a-priori statistical power analysis [10], a sample size of 250 ACS patients and 250 age- and sex- matched healthy subjects, was adequate to evaluate two-sided odds ratios equal to 1.20 , achieving a statistical power $>0.80$ at 0.05 probability level ( $p$-value).

\section{Investigated Parameters}

\section{Socio-Demographic, Clinical, Anthropometric and Lifestyle Characteristics}

Age and sex of the participants were recorded and enabled the matching procedure. Smokers were defined as those who smoked at least 1 cigarette/day and the rest were defined as non-smokers. Physical activity was assessed using the validated for the Greek population International Physical
Activity Questionnaire (IPAQ) index [12, 13]; 3 categories were derived based on the calculated Metabolic Equivalent Task (MET) via the IPAQ, i.e. inactive, moderately active and physically active. Body mass index (BMI) was calculated as weight $(\mathrm{kg})$ divided by standing height $\left(\mathrm{m}^{2}\right)$. Obesity was defined as a BMI $>29.9 \mathrm{~kg} / \mathrm{m}^{2}$.

For all participants a detailed medical history was recorded, including family history of CVD, as well as personal history of hypertension, hypercholesterolemia and diabetes. Patients whose average blood pressure were $\geq 140 / 90 \mathrm{mmHg}$ or were under antihypertensive medication were classified as having hypertension. Hypercholesterolemia was defined as total serum cholesterol levels $>200 \mathrm{mg} / \mathrm{dL}$ or the use of lipid-lowering agents. Diabetes mellitus was defined as fasting blood glucose $>126 \mathrm{mg} / \mathrm{dl}$ or the use of antidiabetic medication.

\section{Assessment of Perceived Stress}

Perceived Stress was assessed using the Perceived Stress Scale, which is a 14-item self-reported questionnaire that evaluates the degree to which individuals appraise their lives as unpredictable, uncontrollable or overloaded, asking participants to recall how stressed they felt in particular situations during the last month [14]. The 14 items were rated from 0 to 4 according to the frequency of feelings experienced by the participant (i.e. never, almost never, sometimes, often or almost always). Total theoretical range of the score is $0-56$. Higher values of the PSS-14 score indicated greater perceived stress. The scale has been validated into Greek by 2 independent groups and has been found reliable and accurate for use $[15,16]$.

\section{Assessment of Depressive Symptomatology and Anxiety}

Depression was evaluated using a translated and validated version of the Zung Depression Rating Scale (ZDRS) [17]. The ZDRS is a self-rating scale consisting of 20 items that cover affective, psychological, and somatic symptoms for the measurement of depression. The individual rates the frequency a symptom is experienced (i.e. little, some, a good part of the time, or most of the time). Total theoretical range of the score is 20-80. Scores of 20-49 are considered normal, 50-59 indicates mild depression, 60-69 moderate to marked depression and 70-80 severe depression.

Anxiety was assessed using the translated and validated version of the Spielberger State-Trait Anxiety Inventory (STAI form Y-2), which is a 20-item self-reported questionnaire evaluating how the respondent feels generally [18]. The 20 items were rated from 1 to 4 according to frequency of their feelings (i.e. almost never, sometimes, often or almost always). Total theoretical range of the score is 20-80. Values of 20-39 suggest low anxiety, 40-59 moderate anxiety and $>59$ severe anxiety [18].

\section{Dietary Assessment}

Dietary habits were assessed through a 90-item, validated semi-quantitative food-frequency questionnaire (FFQ) as previously described [10] and validated [19]. Level of adherence to the Mediterranean diet was evaluated using an 11-item large-scale, composite index, the MedDietScore 
[20]. In brief, for the consumption of foods deemed to be part of the Mediterranean pattern (i.e. those suggested on a daily basis or more than 4 servings/week, such as nonrefined cereals, fruits, vegetables, legumes, olive oil, fish and potatoes) lower scores were assigned when participants reported no, rare or moderate consumption, while higher scores were assigned when the consumption was according to the rationale of the Mediterranean pattern. For the consumption of foods assumed not to be part of the Mediterranean pattern (i.e. meat and meat products, poultry and full fat dairy products), scores were assigned on a reverse scale. For alcohol, score 5 was assigned for consumption of $<3$ wineglasses/day, score 0 for consumption of $>7$ wineglasses/day and scores from 4 to 1 for consumption of $3,4-5,6$ and 7 or 0 wineglasses/day, respectively. The theoretical range of the MedDietScore was between 0-55. Higher values of this diet score indicate greater adherence to the Mediterranean diet. The validation properties of the MedDietScore have been presented elsewhere [20].

\section{Statistical Analysis}

Normally distributed continuous variables (age, BMI, PSS-14, Zung and MedDietScore) are presented as mean \pm standard deviation and categorical variables (sex, smoking, medical history, obesity, physical activity) as frequencies. Normality of the variables was tested using P-P plots. Associations between categorical variables were tested by the calculation of the chi-square test. Comparisons of mean values of normally distributed continuous variables by clinical outcome were performed using the Student's t-test. Correlations between continuous variables were evaluated using the Pearson's $r$ or Spearman rho coefficients. Correlations between continuous and categorical variables were evaluated using the Mann-Whitney test. Estimations of the odds ratios of having ACS according to the level of adherence to PSS-14, the Mediterranean diet, smoking habits, physical activity, diabetes mellitus, hypertension and hypercholesterolemia were performed through logistic regression analysis; results are presented as odds ratios (OR) and the corresponding $95 \%$ confidence intervals (CI). Hosmer-Lemeshow statistic was calculated to evaluate model's goodness-of-fit. All $p$-values were based on twosided hypotheses. SPSS 18.0 software (SPSS Inc., Chicago, Il, USA) was used for all the statistical calculations.

\section{RESULTS}

Table 1 describes the demographic, lifestyle and psychological characteristics in both patients and controls. The mean age of participants did not differ between the 2 groups. The sex distribution did not differ, as well. Mean levels of perceived stress scale (PSS-14, theoretical range 056) differed significantly between patients and controls, with patients reporting $49 \%$ higher score than the healthy participants $(29.6 \pm 8.2 \mathrm{vs} .19 .8 \pm 7.8, \mathrm{p}<0.001)$. The prevalence of clinical characteristics (i.e. obesity, hypertension, hypercholesterolaemia and diabetes mellitus), depression, as well lifestyle variables (i.e. smoking, physical activity, Mediterranean Diet adherence) also differed significantly between the 2 groups (Table 1 ).
PSS-14 score was positively associated with history of hypertension $(\mathrm{U}=23143.0, \mathrm{p}<0.001)$, hypercholesterolemia $(\mathrm{U}=21976.5, \mathrm{p}<0.001)$, diabetes mellitus $(\mathrm{U}=16344.5$, $\mathrm{p}<0.001)$, as well as smoking habits $(\mathrm{U}=22484.0, \mathrm{p}<$ 0.001 ), presence of depressive symptoms as evaluated through the ZDRS scale (rho $=0.576, \mathrm{p}<0.001$ ), and anxiety as evaluated through the STAI scale (rho $=0.589, \mathrm{p}$ $<0.001$ ), and inversely correlated with the MedDietScore that is a proxy of the level of adherence to the Mediterranean $\operatorname{diet}(\mathrm{rho}=-0.219, \mathrm{p}<0.001)$.

However, the aforementioned relationship between perceived stress and likelihood of having ACS may be prone to bias, especially due to the observational design of the study. Thus, multi-adjusted analyses were further performed. Table 2 summarizes the independent factors associated with non-fatal ACS along with their corresponding odds ratios and CIs, as were extracted from the conditional logistic regression analysis. PSS-14 was positively associated (OR: $1.147,95 \%$ CI $1.11-1.18, \mathrm{p}<0.001)$ with the likelihood of having an ACS, even after adjusting for related clinical variables (i.e. hypertension $\mathrm{p}=0.008$, hypercholesterolaemia $\mathrm{p}<0.001$, diabetes mellitus $\mathrm{p}=0.001$ and obesity $\mathrm{p}=0.3$ ), as well as lifestyle characteristics (Mediterranean diet adherence $\mathrm{p}=0.005$, physical activity $\mathrm{p}=0.006$ and smoking $\mathrm{p}<0.001)$. In particular, for each unit increase in the PSS-14 the likelihood of having an ACS increased by $14.7 \%$. Moreover, each unit of increase of the MedDietScore lead to a $44 \%$ lower likelihood of having an ACS. Moreover, physical inactivity almost doubled the likelihood of having an ACS, after various adjustments made (Table 2). STAI and ZUNG were positively associated with the likelihood of having an ACS after adjusting for the aforementioned factors as well (OR: $1.27 \%$ CI 1.20-1.34, p < 0.001 and OR: 1.49 $\%$ CI 1.36-1.63, $\mathrm{p}<0.001$ respectively).

The multivariable analysis was further stratified by sex, by depression and by anxiety levels, since due to collinearity we could not adjust in the same model perceived stress and depression status. Participants whose median ZDRS score was <39 were classified into the low depressive symptomatology group and the rest whose median scores were $>40$ were grouped into moderate-to-high. Participants whose median STAI score was $<39$ were classified into the low anxiety group and the rest whose median score were $>40$ were grouped into moderate-to-high. Odds ratios between men and women were approximately equal (OR for men 1.15 , CI 1.10-1.2, p < 0.001 and OR for women 1.17, CI $1.09-1.25, \mathrm{p}<0.001$, respectively), but the impact of perceived stress was greater in men rather than in women (Wald test value 45.65 vs 18.56 , respectively). When stratifying by level of depression, each unit increase in the PSS-14 was associated with $27 \%$ (\%CI 1.056-1.531) higher odds of having an ACS but only for the participants with low depressive symptomatology. No such significant association was observed in participants with moderate-to-high depressive symptomatology. When stratifying by level of anxiety, each unit increase in the PSS-14 was associated with $12.9 \%$ (\% CI 1.047-1.218) higher odds of having an ACS in the low anxiety group and with $5.5 \%$ (\%CI 1.010-1.103) higher odds of having an ACS in the moderate-to-high anxiety group. 
Table 1. Demographic, lifestyle and clinical characteristics of the study participants $(n=500)$.

\begin{tabular}{|c|c|c|c|}
\hline & ACS patients $n=250$ & ACS controls $n=250$ & $\boldsymbol{P}$ \\
\hline Age & $60 \pm 11$ & $60 \pm 8.6$ & 0.747 \\
\hline \multicolumn{4}{|l|}{ Smoking habits } \\
\hline Non smoker & $80(32 \%)$ & $167(66.7 \%)$ & $<0.001$ \\
\hline \multicolumn{4}{|l|}{ Physical Activity status } \\
\hline Inactive & $99(39.6 \%)$ & $73(29 \%)$ & \multirow[t]{3}{*}{0.002} \\
\hline Moderately active & $88(35.2 \%)$ & $84(33.7 \%)$ & \\
\hline Active & $63(25.2 \%)$ & $93(37.3 \%)$ & \\
\hline Diabetes Mellitus & $103(41.2 \%)$ & $27(10.9 \%)$ & $<0.001$ \\
\hline Body mass index $\left(\mathrm{kg} / \mathrm{m}^{2}\right)$ & $28.3 \pm 4.6$ & $27.1 \pm 3.7$ & 0.003 \\
\hline Non Obese $\left(18.5-29.9 \mathrm{~kg} / \mathrm{m}^{2}\right)$ & $181(72.5 \%)$ & $204(81.7 \%)$ & \multirow[t]{2}{*}{0.016} \\
\hline Obese (>30 kg/m²) & $68(27.5 \%)$ & $46(18.3 \%)$ & \\
\hline MedDietScore (range 0 - 55) & $29.5 \pm 4.5$ & $31.8 \pm 4$ & $<0.001$ \\
\hline Low adherence (0-30) & $146(58.2 \%)$ & $79(31.7 \%)$ & \multirow[t]{2}{*}{$<0.001$} \\
\hline High adherence (31-55) & $104(41.8 \%)$ & $171(68.3 \%)$ & \\
\hline$>40$ & $224(89.6 \%)$ & $67(26.9 \%)$ & $<0.001$ \\
\hline PSS-14 (range 0-56) & $29.6 \pm 8.2$ & $19.8 \pm 7.8$ & $<0.001$ \\
\hline
\end{tabular}

ACS, Acute Coronary Syndromes; ZDRS, Zung Depression Rating Scale; STAI, State-Trait Anxiety Inventory; PSS-14, Perceived Stress Scale-14items.

Furthermore, perceived stress was independently and positively associated with the likelihood of having hypertension (OR: 1.045, \%CI 1.023-1.068, p < 0.001), hypercholesterolaemia (OR: 1.037, \% CI 1.016-1.059, p = 0.001 ) and diabetes mellitus (OR: 1.054, \%CI 1.029-1.081, $\mathrm{p}<0.001$ ), after adjusting for behavioural variables (i.e. adherence to the Mediterranean diet, smoking and physical activity). Further, each unit increase in the PSS-14 was associated with $0.28 \%$ less odds of adopting the Mediterranean diet principles $(0.972, \%$ CI $0.950-0.977, \mathrm{p}=$ 0.025 ) and with $0.47 \%$ higher odds of being a smoker (OR: $1.047, \%$ CI 1.024-1.070, $\mathrm{p}<0.001)$.

\section{DISCUSSION}

In the present study perceived stress was independently and positively associated with first ACS, in a Greek sample of middle-aged men and women. This finding is consistent with previous reports [8]. Moreover, the association of perceived stress with ACS development was similar in both sexes, whereas the presence of depressive symptomatology seems to mask its effect. Anxiety levels did not appear to have an effect on the association of perceived stress with ACS. Further, perceived stress was independently associated with the presence of traditional cardiac risk factors (i.e. hypertension, diabetes mellitus, dietary habits), a finding that confirms previous work $[21,22]$. The retrospective nature of 
Table 2. Results from the multiple conditional logistic regression analysis that was developed to evaluate the likelihood of having an ACS (outcome) according to perceived stress levels among $n=250$ ACS cases and $n=250$ controls.

\begin{tabular}{|c|c|}
\hline Independent predictors & Model \\
\hline \hline MedDietScore (Y/N) & $0.46(0.27,0.79)$ \\
\hline Physical inactivity (Y/N) & $2.53(1.31,4.87)$ \\
\hline Ever smoking (Y/N) & $4.87(2.72,8.69)$ \\
\hline Hypertension (Y/N) & $2.09(1.21,3.63)$ \\
\hline Hypercholesterolaemia (Y/N) & $3.50(2.07,5.93)$ \\
\hline Diabetes mellitus (Y/N) & $3.18(1.65,6.10)$ \\
\hline PSS-14 (per 1/56 unit) & $1.15(1.11,1.18)$ \\
\hline
\end{tabular}

Results are presented as OR (95\% CIs) obtained from multiple conditional logistic regression. PSS-14, Perceived Stress Scale-14 items; ACS, Acute Coronary Syndromes.

the study does not allow the identification of causality; however, the aforementioned findings strengthen the current research towards the stress-cardiovascular risk hypothesis. They also encourage further research to elucidate the role of perceived stress in CVD incidence.

The independent association between ACS incidence and perceived stress poses the question whether this type of stress serves as another possible pathway to CHD. In fact, there is lack of research examining the potential mechanisms linking perceived stress to CHD. However, it is commonly accepted that perceived stress affects CHD outcomes indirectly through adverse changes in health behaviour and in a more direct manner, affecting cardiac risk profile $[21,22]$. For example, perceived stress is associated with a high fat diet, physical inactivity, smoking and drinking $[21,23]$. Apart from behavioural variables, perceived stress is directly linked to diabetes incidence [24] and stressed men and women are more likely to use antihypertensive medication, as well as become overweight in the long run $[21,25]$. What is more, current findings confirm the bidirectional effect of perceived stress on CHD outcomes. In particular, perceived stress was independently and positively associated with the likelihood of having hypertension, hypercholesterolaemia and diabetes mellitus, as well as being a smoker and was negatively associated with the adoption of the Mediterranean diet principles. In another study, perceptions of stressful circumstance were independently associated with greater inflammation and hypercoagulability [26]. The underlying mechanisms that explain the association between perceived stress and such an unfavourable cardiac profile are not completely understood yet. However, previous research on mental stress and its relation to CVD pathogenesis and incidence sheds light on this issue. For example, stress is linked to the pathogenesis of the Metabolic Syndrome, thus it originally promotes insulin resistance and glucose intolerance, as well as adiposity, establishment of hypertension, development of hypercholesterolemia and central obesity, especially, when its effect is prolonged $[27,28]$. The kinetics of the regulation of the Hypothalamic Pituitary Adrenal (HPA) axis seem to play a central role in the connection of stress with such a clustered clinical manifestation [29]. Apart from HPA axis activation and cortisol secretion, other potential mechanisms linking stress to the Metabolic Syndrome serve as several well-known intermediate subclinical markers of CHD progression [4]. Actually, psychological stress is followed by autonomic dysfunction (i.e. parasympathetic activity withdrawal), hemodynamic (i.e. increase in BP and cardiac output), inflammatory (i.e. IL-6 and TNF-a activation) and prothrombotic responses (i.e. monocyte production) $[9,30]$. Prolonged or chronic such effects may increase the risk of CHD development, as well as its progression [31, 32]. Based on the above, further research is needed to explain the architecture of a plausible causality of perceived stress regarding CHD pathogenesis and incidence.

In the present analysis, depressive symptoms masked the effect of perceived stress, whereas the contribution of perceived stress on ACS likelihood remained unaffected in non-depressed individuals. This finding suggests a possible interaction between these 2 psychosocial CHD risk factors, i.e., perceived stress and depression. Indeed, stress is closely related to depression. Actually, chronic stress appears to trigger the development of depression $[33,34]$. For example, chronic activation of the HPA axis, the main neuroendocrine response to stress, is thought to participate in the development of depressive symptoms and depression is characterized by an over-activity of the HPA axis [35]. In other words, depressed individuals are thought to be in a constant state of perceived stress [35]. However, individuals that appraise their lives as stressful chronically are not or they will not become necessarily depressed. Instead, an implicit genetic predisposition to depressive symptoms that interacts with perceived stressful environments may explain why some individuals recover from life stressors and some others do not, developing depression and thus exhibiting them to a certain risk of CVD [36]. Consequently, depression measurement leads to the evaluation of a more prominent psychological manifestation as a result of chronic perception of a stressful life by more susceptible individuals, whereas perceived stress measurement may serve as a more unique and global alternative, that applies to all individuals in life 
periods when depression may or may not exist, or is preparing to manifest.

Apart from depression, perceived stress is related to anxiety as well. Interestingly, the impact of perceived stress remained unaffected among individuals with low and high levels of anxiety, according to our sensitivity analysis. This outcome may imply that albeit an overlap between anxiety and perceived stress [37], these two constructs do maintain a distinct and independent effect on the likelihood of having an ACS. In other words, the present findings confirm that perceived stress may not appear as a hidden underlying dimension of anxiety, but as a single framework with its own risk on CHD incidence [38]. Of note, perceived stress refers to the extent to which individuals appraise their lives as unpredictable, overloaded and uncontrollable [14], whereas anxiety has been documented as the aversive state "resulting from feelings of being unable to predict, control or obtain desired outcomes" [38]. Thus, it appears that perceived stress may serve as one piece in the bigger picture of mental health issues, like, anxiety disorder that presents a documented CVD risk factor as well [39]. Consequently, just like traditional CVD risk factors, psychosocial CVD risk factors play independent and in the same time overlapping roles in CHD pathogenesis, initiating biologic and psychophysiologic processes, as well as behaviours that promote cardiopathogenesis [37].

The aforementioned psychosocial CVD risk factors could be stratified according to the magnitude of ACS risk attributed to each of them. Consequently, in this study, depression emerges as the more prominent psychosocial CVD risk factor, followed by anxiety and perceived stress. This suggested gradient may imply the significance of early treating perceived stress in an attempt to prevent or delay disease progression per se and/or further psychological disorders.

According to our sensitivity analysis, a more pronounced relationship was found between perceived stress and the likelihood of having an ACS in men rather than in women. This discrepancy may lie upon certain differences in stress reactivity and coping that the two genders are thought to exhibit [40]. For example, men appear to cope with stress in a more rational, detached and not so emotion-focused way [40]. They are typically more assertive, hostile and susceptible to aggressive and risk taking behaviours [41]. In contrast, women typically tend to cope with stress more emotionally and tend to buffer its effect through social networking. More specific, females are more likely to mobilize social support from other females and become satisfied with the support they receive, when defending to stressors. This desire to affiliate with others under stressful conditions is substantially more marked among females than among males and this may be the primary gender difference in adult human behavioural responses to stress [40]. The way men tend to defend to stressors follows the traditional "fight or flight" response, whereas alternatively women exhibit a more of a "tend and befriend" behavioural pattern as a response to stressors [40]. The "fight or flight" behavioural response may account for the stronger stress impact on the likelihood of having an ACS in males rather in females. In fact, there is evidence supporting greater neuroendocrine stress responsiveness in males compared with women [42] and that this has been suggested to be associated with the pathogenesis of CVD [43].

\section{LIMITATIONS}

The retrospective nature of the present study does not allow for causal inferences and predisposes to systematic selection and/or recall bias. To minimize selection bias cases with a first ACS event were only enrolled. To minimize recall bias accurate and detailed data from all participants during the first 3 days of hospitalisation were obtained. Perceived stress and depression status were evaluated through validated to the referent population's questionnaires. However, measurements of psychological characteristics usually succumb to over- or under- reporting and does not always resemble the accuracy of measuring specific psychological manifestations (i.e. depression disorder subsets). Furthermore, an effort was made to evaluate participants' levels of perceived stress at least 2 months before the ACS outcome, in order to avoid over-estimation. Regarding dietary evaluation, an FFQ was administered and applied by trained dieticians through face-to-face interviews in an effort to reduce inaccuracies of dietary reporting. Additionally, an effort was given to retrieve accurate information from participants' medical records, as well as their relatives regarding the onset of CVD risk factors and smoking. Finally, the inclusion of patients and controls from only 2 regions may limit the generalization of the findings to the whole country. Nevertheless, it should be noted that Athens is the capital of Greece and Larisa the metropolitan city in central Greece. Thus, both represent a vast majority of the Greek urban and rural population.

\section{CONCLUSION}

The potential identification of perceived stress as a distinct psychosocial ACS risk factor bears certain implications for future preventive measures in susceptible populations. In particular, early recognition and treatment of perceived stress may lead to improvements in lifestyle habits, as well as modification of the potential adverse physiological changes of stress and, thus, retardation of CHD progress. Additionally, early treatment of perceived stress could prevent or reduce depressive symptoms, as well as anxiety disorder and, thus, further modify CVD risk [41]. Consequently, perceived stress should not remain unrecognized in such populations.

\section{CONFLICT OF INTEREST}

The authors declare that they have no conflict of interest.

\section{AKNOWLEDGMENTS}

We thank our field investigators: Julia DeVita, Georgio Karayanni, Emmanuela Griva, Asimenia Kouroupi, Evaggelia Nanou, Konstantino Syriano, Dionysia Voutsa and Nikolao Zaxaro.

\section{REFERENCES}

[1] Perk J, De Backer G, Gohlke H, et al. European guidelines on cardiovascular disease prevention in clinical practice (version 
2012) The fifth joint task force of the European society of cardiology and other societies on cardiovascular disease prevention in clinical practice (constituted by representatives of nine societies and by invited experts). Int J Behav Med 2012; 19: 403-88.

[2] Andrikopoulos G, Richter D, Sakellariou D, et al. High prevalence and diminished awareness of overweight and obesity in a Mediterranean population. An alarming call for action. Open Cardiovasc Med J 2012; 6: 141-6.

[3] Von Känel R. Psychosocial stress and cardiovascular risk: current opinion. Swiss Med Wkly 2012; 142: W13502.

[4] Coughlin SS. Post-traumatic stress disorder and cardiovascular disease. Open Cardiovasc Med J 2011; 5: 164-70.

[5] Chandola T, Britton A, Brunner E, et al. Work stress and coronary heart disease: what are the mechanisms? Eur Heart J 2008; 29: 6408.

[6] Eaker ED, Sullivan LM, Kelly-Hayes M, D'Agostino RB Sr, Benjamin EJ. Marital status, marital strain, and risk of coronary heart disease or total mortality: the Framingham offspring study. Psychosom Med 2007; 69: 509-13.

[7] Rosengren A, Hawken S, Ounpuu S, et al. Association of psychosocial risk factors with risk of acute myocardial infarction in 11,119 cases and 13, 648 controls from 52 countries (the INTERHEART study): case - control study. Lancet 2004; 364: 953-62.

[8] Katsarou AL, Triposkiadis F, Panagiotakos D. Perceived stress and vascular disease: Where are we now? Angiology 2012; doi: $10.1177 / 0003319712458963$

[9] Richardson S, Shaffer JA, Falzon L, Krupka D, Davidson KW, Edmondson D. Meta-analysis of perceived stress and its association with incident coronary heart disease. Am J Cardiol 2012; 110: $1711-6$.

[10] Kastorini C-M, Milionis HJ, Goudevenos JA, Panagiotakos DB. Modeling the role of dietary habits and eating behaviors on the development of acute coronary syndrome or stroke: aims, design, and validation properties of a case-control study. Cardiol Res Pract 2010; 2011: 313948

[11] Thygesen K, Alpert JS, White HD. Universal definition of myocardial infarction. J Am Coll Cardiol 2007; 50: 2173-95.

[12] Craig CL, Marshall AL, Sjostrom M, et al. International physical activity questionnaire: 12-country reliability and validity. Med Sci Sports Exerc 2003; 35: 1381-95.

[13] Papathanasiou G, Georgoudis G, Papandreou M, et al. Reliability measures of the short International Physical Activity Questionnaire (IPAQ) in Greek young adults. Hellenic J Cardiol 2009; 50: 283-94.

[14] Cohen S, Kamarck T, Mermelstein R. A global measure of perceived stress. J Health Soc Behav 1983; 24: 385-96.

[15] Katsarou A, Panagiotakos D, Zafeiropoulou A, et al. Validation of a Greek version of PSS-14; a global measure of perceived stress. Cent Eur J Public Health 2012; 20: 104-9.

[16] Andreou E, Alexopoulos E, Lionis C, et al. Perceived stress scale: reliability and validity study in greece. Int $\mathrm{J}$ Environ Res Public Health 2011; 8: 3287-98.

[17] Fountoulakis K, Iacovides A, Samolis S, et al. Reliability, validity and psychometric properties of the Greek translation of the zung depression rating scale. BMC Psychiatry 2001; 1: 6.

[18] Fountoulakis KN, Papadopoulou M, Kleanthous S, et al. Reliability and psychometric properties of the Greek translation of the statetrait anxiety inventory form Y: preliminary data. Ann Gen Psychiatry 2006; 5: 2.

[19] Bountziouka V, Bathrellou E, Giotopoulou A, et al. Development, repeatability and validity regarding energy and macronutrient intake of a semi-quantitative food frequency questionnaire:
Methodological considerations. Nutr Metab Cardiovasc Dis 2012; 22: 659-67.

[20] Panagiotakos DB, Pitsavos C, Stefanadis C. Dietary patterns: a Mediterranean diet score and its relation to clinical and biological markers of cardiovascular disease risk. Nutr Metab Cardiovasc Dis 2006; 16: 559-68.

[21] Rod NH, Grønbaek M, Schnohr P, Prescott E, Kristensen TS Perceived stress as a risk factor for changes in health behaviour and cardiac risk profile: a longitudinal study. J Intern Med 2009; 266: 467-75.

[22] Adler N, Matthews K. Health psychology: why do some people get sick and some stay well. Annu Rev Psychol 1994; 45: 229 -59.

[23] Ng DM, Jeffery RW. Relationships between perceived stress and health behaviors in a sample of working adults. Health Psychol 2003 ; 22 : 638-42.

[24] Novak M, Björck L, Giang KW, Heden-Ståhl C, Wilhelmsen L, Rosengren A. Perceived stress and incidence of Type 2 diabetes: a 35-year follow-up study of middle-aged Swedish men. Diabet Med 2012; 30: 8-16.

[25] Kato M, Noda M, Inoue M, Kadowaki T, Tsugane S; JPHC Study Group. Psychological factors, coffee and risk of diabetes mellitus among middle-aged Japanese: a population-based prospective study in the JPHC study cohort. Endocr J 2009; 56: 459-68.

[26] Jain S, Mills PJ, von Känel R, Hong S, Dimsdale JE. Effects of perceived stress and uplifts on inflammation and coagulability. Psychophysiology 2007; 44: 154-60.

[27] Chrousos GP. The role of stress and the hypothalamic-pituitaryadrenal axis in the pathogenesis of the metabolic syndrome: neuroendocrine and target tissue-related causes. Int J Obes Relat Metab Disord 2000; 24: 50-5.

[28] Anagnostis P, Athyros VG, Tziomalos K, Karagiannis A, Mikhailidis DP. Clinical review: the pathogenetic role of cortisol in the metabolic syndrome: a hypothesis. J Clin Endocrinol Metab 2009; 94: 2692-701.

[29] Björntorp P, Rosmond R. The metabolic syndrome--a neuroendocrine disorder? Br J Nutr 2000; 83: 49-57.

[30] Steptoe A, Brydon L. Emotional triggering of cardiac events Neurosci Biobehav Rev 2009; 33: 63-70.

[31] Triposkiadis F, Starling RC, Stefanadis C. Diagnosis and management of chronic coronary artery disease. Curr Cardiol Rev 2007; 3: 221-31

[32] Onat A, Can G, Hergenç G, Küçükdurmaz Z, Uğur M, Yüksel H. High absolute coronary disease risk among Turks: involvement of risk factors additional to conventional ones. Cardiology 2010; 115 : 297-306.

[33] Joynt KE, Whellan DJ, O'Connor CM. Depression and cardiovascular disease: mechanisms of interaction. Biol Psychiatry 2003; 54: 248-61.

[34] Rumsfeld JS, Ho MP. Depression and cardiovascular disease: a call for recognition. Circulation 2005; 111: 250-3.

[35] Pariante CM. Depression, stress and the adrenal axis J Neuroendocrinol 2003; 15: 811-2.

[36] Caspi A, Sugden K, Moffitt TE, et al. Influence of life stress on depression: moderation by a polymorphism in the 5-HTT gene. Science 2003; 301: 386 -9.

[37] Suls J, Bunde J. Anger, anxiety, and depression as risk factors for cardiovascular disease: the problems and implications of overlapping affective dispositions. Psychol Bull 2005; 131: 260-300.

[38] Dietrich M, Verdolini AK, Gartner-Schmidt J, Rosen CA. The frequency of perceived stress, anxiety, and depression in patients with common pathologies affecting voice. J Voice 2008; 22: $472-88$. 
[39] Olafiranye O, Jean-Louis G, Magai C, et al. Anxiety and cardiovascular symptoms: the modulating role of insomnia. Cardiology 2010; 115: 114-9.

[40] Schmitt DP, Realo A, Voracek M, Allik J. Why can't a man be more like a woman? sex differences in big five personality traits across 55 cultures. Pers Soc Psychol 2008; 94: 168-82.
[41] Wang J, Korczykowski M, Rao H. Gender difference in neural response to psychological stress. Soc Cogn Affect Neurosci 2007; 2: 227-39.

[42] Kudielka BM, Kirschbaum C. Sex differences in HPA axis responses to stress: a review. Biol Psychol 2005; 69: 113-32.

[43] De Backer G, Graham I, Cooney MT. Do novel biomarkers add to existing scores of total cardiovascular risk? Eur J Prev Cardiol 2012; 19:14-1.

Received: March 02, 2014

Revised: May 15, 2014

Accepted: June 02, 2014

(C) Katsarou et al.; Licensee Bentham Open.

This is an open access article licensed under the terms of the Creative Commons Attribution Non-Commercial License (http://creativecommons.org/licenses/ by-nc/3.0/) which permits unrestricted, non-commercial use, distribution and reproduction in any medium, provided the work is properly cited. 